\title{
THE ENDS OF PRODUCT MANIFOLDS
}

\author{
DENNIS C. HASS
}

\begin{abstract}
In this paper we provide simple point set properties which characterize the $m$-spheres $S^{m}$, open $m$-cells $E^{m}$, closed cells $I^{m}$, and annuli $A^{m}=[0,1) \times S^{m-1}$. It is important to notice that the Poincaré conjecture is not used in dimension 3 or 4 .
\end{abstract}

In this paper we provide simple point set properties which characterize $m$-spheres $S^{m}$ and open $m$-cells $E^{m}$ among $m$-manifolds. Accepting these two main theorems, we establish corollaries which characterize closed cells $I^{m}$ and annuli $A^{m}=[0,1) \times S^{m-1}$. Then we prove the two main theorems. It is important to note that the Poincaré conjecture is not used in dimension 3 or 4 .

In the following $M$ will mean an $m$-manifold, for $m \geqq 2$, and $P$ is a point in $M$. We will always be asking for geometric consequences of supposing that a certain subset of $M$ (such as $M-P$ ) is a product $A \times B$ of topological spaces where neither $A$ nor $B$ is a single point. Recall that such factors $A, B$ are necessarily generalized manifolds in the sense of Wilder ([1], [2]). We will repeatedly use the fact that $A$ or $B$ is therefore a manifold if its dimension is $\leqq 2[1]$.

1. The proofs of the first two theorems are deferred until the next section.

THEOREM 1. $M=E^{m}$ is the only connected noncompact m-manifold such that $M-P$ is a product space.

THEOREM 2. $M=S^{m}$ is the only connected compact m-manifold such that $M-P$ is a product space.

COROLlaRY 3. It is not necessary in Theorem 2 to assume that $M$ is connected.

Proof. Let $N$ be the component of $M$ with $P$ in $N$. By Theorem 2, $N=S^{m}$. Now let $M-P=X \times Y$ and $X_{0}, Y_{0}$ be the components of $X$ and $Y$

Received by the editors August 22, 1971.

AMS 1970 subject classifications. Primary 54F65, 55A40, 57A15, 57B99; Secondary 54E45, 57A05, 57A10, 57C99, 57D99.

Key words and phrases. Topological manifold, generalized manifold, spheres, cells, annulus, product space, ends, Poincaré theorem, residual set.

(c) American Mathematical Society 1972 
for which $N-P=X_{0} \times Y_{0}$. Then $X_{0}$ and $Y_{0}$ are contractible (hence noncompact). If $X_{1}$ is another component of $X$, then $X_{1} \times Y_{0}$ is a noncompact component of $M$. This contradiction means $X$ is connected. Similarly $Y$, and hence $M$, is connected. Thus $M=N=S^{m}$.

Without the connectivity, Theorem 1 fails, even for $m=2$. There are two distinct 2-manifolds $M$ and $M_{1}$ such that $M-P=M_{1}-P_{1}=$ $\left(E^{1} \cup S^{1}\right) \times E^{1}$ is a product of $E^{1}$ by a disjoint union.

We now turn our attention to manifolds $M$ with boundary $b M$ and interior $\operatorname{Int}(M) . P$ will always be a point in the interior of $M$ and $Q$ a point in $b M$.

THEOREM 4. These conditions are equivalent for a connected m-manifold with $b M=S^{m-1}$ :

(a) $M=I^{m}$.

(b) Int $M$ is a product space and $M$ is compact.

(c) $M$ is a compact product space.

(d) Int $M-P$ is a product space.

(e) $M-Q$ is a product space and $M$ is compact.

Proof. (a) clearly implies the rest of the list. Each of (e) and (c) implies (b). We will show (b) implies (a). Let $N=M / b M$ be the quotient space. Then $N-(b M / b M)$ is Int $M$, which is a product space. Since $N$ is compact, $N=S^{m}$ by Theorem 2 . Now Int $M=E^{m}$ which implies (a) by Schoenflies [3]. That (d) implies (a) follows from Theorem 1.

In condition (d) compactness is a consequence rather than a hypothesis. According to Theorem 7, below, this is often true of condition (e) as well.

THEOREM 5. These conditions are equivalent for a connected m-manifold $M$ with $b M=S^{m-1}$ :

(a) $M=[0,1) \times S^{m-1}=A^{m}$.

(b) Int $M$ is a product space and $M$ is not compact.

(c) $M$ is a noncompact product space.

Proof. Clearly (a) implies (c) and (c) implies (b). We shall show that (b) implies (a) by forming the manifold $N=M / b M$. Since $N-(b M / b M)=$ Int $M$ is a product space, $N=E^{m}$ by Theorem 1 . The theorem follows.

The next theorem fully describes closed cells and annuli entirely in point set terms.

THEOREM 6. Let $M$ be a connected m-manifold with compact boundary bM. If each of $M$ and $b M-Q$ is a product space, then $M$ is either $I^{m}$ or $[0,1) \times S^{m-1}$.

Proof. By Corollary $3, b M$ is $S^{m-1}$. If $M$ is compact, $M=I^{m}$ by Theorem 4(c). If not, then $M=A^{m}$ by Theorem 5(c). 
For most $m$, we have two other characterizations of $I^{m}$. Whenever $X$ is a generalized manifold, let $b X$ denote its boundary.

THEOREM 7. If $M-Q=X \times Y$ is a product space with $b X=\varnothing$, then

(a) the double $2 M$ is $S^{m}$, so $M$ is compact;

(b) $b M$ is a homotopy $(m-1)$-sphere;

(c) Int $M=E^{m}$;

(d) $M=I^{m}$, unless $m=4$ or 5 ; and

(e) $M-Q=(b M-Q) \times[0,1)$.

Proof. $2 M-Q=2(X \times Y)=X \times 2 Y$, because $b X=\varnothing$ and because $Q$ is in $b M$. Thus, either $2 M=S^{m}$ by Theorem 2 , or $2 M=E^{m}$ by Theorem 1 . In the latter case we use the proof of Theorem 1 to conclude further that $X$ is a compact (generalized) homotopy $(m-1)$-sphere and that $2 Y=E^{\mathbf{1}}$. Now, $Y=[0,1)$ and $M-Q=X \times[0,1)$. Since $b M-Q=X$ and since $X$ is compact and $b M-Q$ is not, we have reached a contradiction. Thus, $2 M=S^{m}$ as stated.

If $\operatorname{dim} Y>1$, then $b M-Q=b(M-Q)=b(X \times Y)=X \times b Y$ is a product. By Theorem 2, $b M=S^{m-1}$. By Theorem $4(\mathrm{~b}), M$ is $I^{m}$, completing the theorem.

Thus, we let $\operatorname{dim} Y=1$. Since $E^{n}=S^{m}-Q=2 M-Q=X \times 2 Y$, we have $2 Y=E^{1}$ and $Y=[0,1)$ and $X$ is contractible. Further, $b M-Q=b(M-Q)=$ $X$. This means $b M$ is a homotopy sphere. Unless $m=4$ or $5, b M=S^{m-1}$ and $M=I^{m}$ by Theorem 4(b). Finally, $E^{m}=S^{m}-Q=2 M-Q=X \times 2 Y=$ $X \times E^{1}=$ Int $M$, completing the theorem.

THEOREM 8. If $b M$ is compact and Int $M-P=X \times Y$ is a product space, then

(a) Int $M=E^{m}$, so $M$ is compact;

(b) $b M$ is a homotopy sphere;

(c) $2 M=S^{m}$; and

(d) $M=I^{m}$, unless $m=4$ or 5 .

Proof. (a) follows from Theorem 1 and implies (b). (c) holds because $2 M$ is the union of two $m$-cells. (d) follows from the Poincare conjecture in the correct dimension.

2. In order to prove the two main theorems, we begin by studying the ends of open generalized manifolds. See Siebenmann [4] for a discussion of ends and Wilder [1, Chapter 8] for a definition of generalized manifolds.

Let $M$ be a $j$-connected generalized manifold and let $M$ have an isolated end $E$ which is $k$-connected (i.e. $E$ has small $k$-connected neighborhoods). Although generally no relationship exists between $j$ and $k$, it is instructive to examine the following class of doughnut-like manifolds. 
Let $N=S^{m+1} \times I^{n+2}$. Now, Int $N=S^{m+1} \times E^{n+2}$ is $m$-connected and has one isolated end whose groups are those of $b N=S^{m+1} \times S^{n+1}$. Thus, $j=m$, and $k=\min \{m, n\}$. It follows from Corollary 11 below that, just as in this example, it is always true that $j \geqq k$ for such products. Proofs of the next three propositions appear in the author's dissertation [5]. Let $M=A \times B$ be an open generalized $m$-manifold.

LEMMA 9. If neither $A$ nor $B$ is compact, then $M$ has exactly one end.

Let $M=A \times B$ have at least one isolated end $E$.

LEMMA 10. If E has a $k$-connected neighborhood and if $A$ is not compact, then $B$ is k-connected, even if $B$ is not compact.

The import here is that $B$ is a retract of every such neighborhood.

CoRollary 11. If $M$ is $k$-connected at $E$, and if $A$ is not compact, then $B$ is $k$-connected. Thus, if neither $A$ nor $B$ is compact, then each of $M, A$ and $B$ is $k$-connected. That is, $j \geqq k$.

LEMMA 12. If $K \subset E^{1} \times B$ is compact, then there is another copy of $K$ in $E^{1} \times B$ disjoint from $K$.

Proof. Since $K$ is compact, there is an $r$ in $E^{1}$ such that $K \subset(-r, r) \times B$. The homomorphism $h$ given by $h(x, b)=(x+3 r, b)$ is a translation which moves $K$ disjoint from itself.

THEOREM 13. If $M$ is an m-manifold with $P$ in Int $M$, and if $M-P=$ $A \times B$ is a product space, then $M$ is $(m-2)$-connected. Moreover, if $B$ is compact, then $M-P=E^{1} \times B$.

Proof. By general position (in a coordinate chart about $P$ ), the homotopy groups of $M$ and $M-P$ agree up to dimension $m-2$. If neither $A$ nor $B$ is compact, then we are done by Corollary 11 . Let $B$ be compact. Using Poincaré duality on $B, H_{i} B=Z_{2}$ for $i=\operatorname{dim} B$. From Corollary 11 , $B$ is $(m-2)$-connected; that is, $H_{n} B=0$ for $n \leqq m-2$. Thus $\operatorname{dim} B=m-1$ and $\operatorname{dim} A=1$. This means $A=E^{1}$ and $M-P=E^{1} \times B$. Now, $M-P$ is $(m-2)$-connected, because $B$ is.

Now we are equipped to prove the two main theorems. Let $P$ be in $M$.

Proof of Theorem 1. By Lemma 9, if neither $A$ nor $B$ is compact, then $M-P$ has just one end. Since this makes $M$ compact, we may instead assume that $B$ is compact.

By Theorem 13, $M-P=E^{1} \times B$. This makes $M$ the open cone $C B$ on $B$. Thus $M=E^{m}$ by Brown's open star theorem [6].

Proof of Theorem 2. By Theorem 13, $M$ is $(m-2)$-connected. Using the Poincaré theorem, we find $M=S^{m}$ for $m \geqq 5$. Thus, let $m \leqq 4$. From 
Theorem 13, we find that if $B$ is compact, $M-P=E^{1} \times B$ has two ends. Since this denies that $M$ is compact, we may assume that neither $A$ nor $B$ is compact. It follows that each of $M-P, A$, and $B$ is contractible. If $m=2, A=B=E^{1}$ so $M=S^{2}$. If $m=3, A=E^{1}, B=E^{2}$ or vice-versa, so $M=S^{3}$. Let $m=4$. If $\operatorname{dim} B=2$, then $A=B=E^{2}$, so $m=S^{4}$. We have left only the case $m=4$ and $\operatorname{dim} B=3$. In this case $A=E^{1}$ and $M-P=E^{1} \times B$. We decompose $M=e^{4} \cup K$ as a disjoint union of an open 4-cell $e^{4}$ and a compact residual set $K$ with $P$ not in $K$. Using Lemma 12 to translate $K$ in $E^{1} \times B$, we find that $M$ is a union of two open cells, and, hence, $M=S^{4}$; see [7].

ACKNOWLEDGEMENT. The author wishes to thank Dr. Patrick $\mathrm{H}$. Doyle for his help and Michigan State University for its support during research on this topic.

\section{BIBLIOGRAPHY}

1. R. L. Wilder, Topology of manifolds, Amer. Math. Soc. Colloq. Publ., vol. 32, Amer. Math. Soc., Providence, R.I., 1949. MR 10, 614.

2. F. Raymond, Separation and union theorems for generalized manifolds with boundary, Michigan Math. J. 7 (1960), 7-21. MR 22 \#11388.

3. M. Brown, A proof of the generalized Schoenflies theorem, Bull. Amer. Math. Soc. 66 (1960), 74-76. MR 22 \#8470b.

4. L. C. Siebenmann, The obstruction to finding a boundary for an open manifold of dimension greater than five, Thesis, Princeton University, Princeton, N.J., 1965.

5. D. C. Hass, The ends of a product manifold, Thesis, Michigan State University, East Lansing, Mich., 1970.

6. B. Mazur, The method of infinite repetition in pure topology. I, Ann. of Math (2) 80 (1964), 201-226. MR 29 \#6477.

7. P. H. Doyle and J. G. Hocking, $A$ decomposition theorem for n-dimensional manifolds, Proc. Amer. Math. Soc. 13 (1962), 469-471. MR 25 \#4514.

Department of Mathematics, Randolph-Macon Woman's College, Lynchburg, VIRGINIA 24504 